\title{
Significance of serine threonine tyrosine kinase 1 as a drug resistance factor and therapeutic predictor in acute leukemia
}

\author{
SHINYA NIRASAWA, DAISUKE KOBAYASHI, TAKASHI KONDOH, KAGEAKI KURIBAYASHI, \\ MAKI TANAKA, NOZOMI YANAGIHARA and NAOKI WATANABE
}

Department of Clinical Laboratory Medicine, Sapporo Medical University School of Medicine, Sapporo 060-8543, Japan

Received June 13, 2014; Accepted July 30, 2014

DOI: 10.3892/ijo.2014.2633

\begin{abstract}
Alterations in the mRNA expression or the mutation of previously reported tyrosine kinases have been detected only in a limited number of patients with acute leukemia. In this study, we examined whether the widely expressed serine threonine tyrosine kinase 1 (STYK1)/novel oncogene with kinase domain (NOK) acts as a drug resistance factor in acute leukemia. The transfection of leukemic HL-60 cells with an STYK1 expression vector resulted in the resistance to doxorubicin and etoposide and decreased drug-induced caspase- $3 / 7$ activity and sub-G1 population. To investigate the mechanism of STYK1-induced drug resistance, microarray analysis was performed using HL-60 cells transfected with control or STYK1 expression vectors. Three tyrosine kinases (EphA4, FLT4 and STK31), two NF- $\kappa \mathrm{B}$ inducers (MAPK4 and TNF-RSF11A), and two genes essential for stem cell replication (SALL4 and NOV) were identified as novel STYK1-induced genes. In addition to the data using cell line, a comparison of the leukemic patients who did and did not respond to therapy revealed that STYK1 expression before therapy was significantly higher in the non-responder group compared with the group that responded completely. These results suggest that STYK1 is a novel drug resistance factor and could be a predictor of the therapeutic response in acute leukemia.
\end{abstract}

\section{Introduction}

Anticancer drug resistance is evoked by various mechanisms, but there is no universal marker that plays a role in drug resistance and that is useful to predict the therapeutic effect in different disease types. To characterize such markers, it is important to identify candidates that are expressed consti-

Correspondence to: Dr Naoki Watanabe, Department of Clinical Laboratory Medicine, Sapporo Medical University School of Medicine, South-1, West-16, Chuo-Ku, Sapporo 060-8543, Japan

E-mail:watanabn@sapmed.ac.jp

Key words: serine threonine tyrosine kinase 1, mRNA expression, chemo-resistance, prediction, acute leukemia tutively in patients before therapy and that promote natural resistance.

The overexpression and mutation of various tyrosine kinases contributes to the development of acute leukemia. For example, the overexpression or mutation of tyrosine kinases, including Flt3, c-kit, platelet-derived growth factor receptor and Bcr-Abl has been reported (1-3). Once these tyrosine kinases are activated, they transmit major molecules such as phosphatidylinositol 3-kinase (PI3K) and mitogen-activated protein kinase (MAPK) (1-3). This not only induces proliferation of leukemia cells, but also renders them resistant to various anticancer drugs. Therefore, several kinase inhibitors are used clinically and some were reported to serve as predictive factors for the patient response to treatment or prognosis (4-6). However, these abnormalities are detected only in a limited number of patients with acute leukemia. Furthermore, energy-dependent, rapid drug efflux and multidrug-resistance molecules are the major factors for the resistance of several leukemic cells; however, drug retention does not always correlate with cytotoxicity and these molecules are not always expressed before therapy.

We recently reported that serine threonine tyrosine kinase 1 (STYK1)/novel oncogene with kinase domain (NOK), a receptor protein tyrosine kinase (RPTK)like protein, is widely expressed in patients with leukemia (7). We also demonstrated that STYK1 expression was decreased after chemotherapy in all patients, and was important in the proliferation of leukemia cells. STYK1 was reported to be a tumorigenesis-inducing factor by Liu et al (8). STYK1 overexpression results in the growth factor-independent proliferation of murine bone-marrow-derived lymphoid BaF3 cells and surface adhesion-independent growth and colony formation in NIH3T3 and BaF3 cells (8). STYK1 shares $20-30 \%$ homology with members of the fibroblast growth factor receptor and platelet-derived grow th factor receptor families (8). RPTKs usually bind to specific ligands to stimulate tyrosine phosphorylation and growth signal transduction. Interestingly, $S T Y K 1$ has a single putative transmembrane domain and an intracellular tyrosine kinase domain, but it lacks an extracellular domain. Therefore, $S T Y K 1$ expression may itself trigger self-phosphorylation and transmit growth signals without ligand binding. PI3K and MAPK are reported as activated growth signals by STYK1, suggesting that these signals might induce the expression of various molecules 
such as inhibitor of apoptosis proteins (IAPs) and NF- $\kappa \mathrm{B}$ signals to stimulate resistance against anticancer drugs. Based on these observations, we hypothesized that the widely expressed STYK1 may play key roles in the drug resistance of hematopoietic malignancies. However, STYK1 expression has not yet been reported to act as a resistance factor in leukemic cells; therefore, whether its expression is related to therapeutic outcome in patients with acute leukemia remains unclear.

In this study, to determine whether STYK1 functions as a resistance factor against anticancer drugs, we transfected cells with either a control or STYK1 expression vector and compared drug sensitivity and the activation of signaling pathways. We next examined STYK1 mRNA expression levels in patients with acute leukemia and compared its expression in two groups with completely different therapeutic outcomes. To ensure the applicability of the mRNA expression measurements to clinical practice, we used peripheral blood and bone marrow blood samples without purifying blasts.

\section{Materials and methods}

Cell culture and anticancer drugs. The human myelogenous leukemia cell lines HL-60 and K562 were obtained from the American Type Culture Collection (Manassas, VA, USA). The cells were cultured in RPMI-1640 (BioWhittaker, Walkersville, MD, USA) supplemented with $10 \%$ heat-inactivated fetal bovine serum (FBS; Invitrogen, Carlsbad,CA,USA) at $37^{\circ} \mathrm{C}$ in a humidified atmosphere of $5 \% \mathrm{CO}_{2}$. Doxorubicin and etoposide were purchased from Sigma-Aldrich (St. Louis, MO, USA).

Patients and samples. A total of 34 peripheral or bone marrow blood samples were examined. Fresh peripheral blood samples from 22 patients who had been diagnosed with acute leukemia were obtained at Sapporo Medical University Hospital. Fresh bone marrow blood samples were also obtained from 12 patients with acute leukemia. Ten patients with acute lymphoblastic leukemia, 20 with acute myeloid leukemia and 4 with acute mixed-lineage leukemia were assessed. A total of 25 patients were newly diagnosed and 9 were relapsed cases. After informed consent was obtained, blood samples were freshly prepared immediately before therapy, hemolyzed using a lysis reagent (Ortho Diagnostic Systems, Tokyo, Japan), and washed with phosphate-buffered saline lacking magnesium and calcium. Total RNA was then extracted from the washed cells.

Transfection. The STYK1 expression vector (pCMV6STYK1) was purchased from Origene Technologies, Inc. (Rockville, MD, USA) and large-scale preparation was performed using competent cells. The control vector (pCMV6Mock) was constructed by digesting the parental vector with AsiSI (SfaAI) and MluI to remove the STYK1 coding region. Both plasmids were transfected into HL-60 cells using the Nucleofector ${ }^{\circledR}$ II device and the Cell Line Nucleofector ${ }^{\circledR}$ Kit V (Amaxa Inc., Gaithersburg, MD, USA) according to the manufacturer's instructions. Transfected cells were then cultured in media containing $800 \mu \mathrm{g} / \mathrm{ml}$ of G418 sulfate (geneticin, EMD Chemicals, Inc., San Diego, CA, USA) for 14 days.
Microarrays. Total RNA was prepared from transfected cells, and then subjected to industrial analysis. Quality control check and global gene expression profiling was performed by TakaraBio. Inc. (Otsu, Japan) using SurePrint G3 Human GE 8x60 K Microarrays (both from Agilent Technologies, Santa Clara, CA, USA) following the Agilent one-color microarray-based gene expression analysis protocol. The slides were scanned using an Agilent Technologies Microarray Scanner, and the images were processed using Agilent Feature Extraction software, version 10.7.3.1.

RNA extraction and quantification of STYK1 mRNA expression. The expression of STYK1 mRNA was determined by performing qRT-PCR on an ABI PRISM 7700 sequence detection system (Applied Biosystems, Foster City, CA, USA). Total RNA was isolated, and the concentration was determined using the GeneQuant DNA/RNA Calculator (Amersham Pharmacia Biotech, Uppsala, Sweden). The gene-specific primers and fluorescent hybridization probes used for quantitative PCR were as follows: STYK1 forward primer, 5'-CAT CTT TCG AGC CAA TAT GAA CAC-3'; reverse primer, 5'-TGG AAT TGG ATT CGC CCT AA-3'; and probe, 5'-(FAM) CCA GCT GGG CTC CAT GAG GTA CAA GAT (TAMRA)-3'. Quantitative RT-PCR was performed using the TaqMan One-Step RT-PCR Master Mix Reagents kit (Applied Biosystems). To compare the STYK1 mRNA expression between different samples, the specific mRNA was normalized to that of $18 \mathrm{~S}$ ribosomal RNA (rRNA) to obtain a ratio. The $18 \mathrm{~S}$ rRNA expression was determined using TaqMan Ribosomal RNA control reagents (Applied Biosystems), according to the protocol provided by the manufacturer. For each experiment, a calibration curve was prepared using control RNA from K562 cells. Briefly, a computer algorithm was used to analyze the emission of reporter dye and quenching dye emission during PCR amplification, and the intensity of the fluorescence signals from each PCR cycle was detected. The amplification curves obtained from serial dilutions of control RNA were prepared, and the optimal signal intensity (threshold) was selected manually in the exponential phase of the curves. The PCR cycle number (threshold cycle, $\mathrm{C}_{\mathrm{T}}$ ) at each concentration of starting RNA was determined to draw the calibration curve, which was constructed as a xy plot [with the log of the input amount ( $\log$ ng of starting total RNA) as $\mathrm{x}$, and $\mathrm{C}_{\mathrm{T}}$ as $\mathrm{y}$. The expression of the target mRNA in the unknown samples was determined from the $C_{T}$ value. A negative control reaction that lacked template was included in each experiment.

Immunocytochemistry. STYK1 protein expression was assessed by immunocytochemistry using anti-STYK1 antibody. Briefly, cells were attached to glass slides by using CytoSpin (1,000 rpm for $1 \mathrm{~min})$, and then fixed immediately using $4 \%$ paraformaldehyde for $15 \mathrm{~min}$ at room temperature (RT). Cells were then incubated with primary antibody (rabbit monoclonal anti-STYK1, Abgent, Inc., San Diego, CA, USA) for $16 \mathrm{~h}$ at $4^{\circ} \mathrm{C}$. Non-specific binding was removed by washing with phosphate-buffered saline (PBS), and cells were then incubated with Alexa Fluor 488-labeled anti-rabbit IgG. Cells were counterstained with Hoechst 33342 for nuclear staining. 


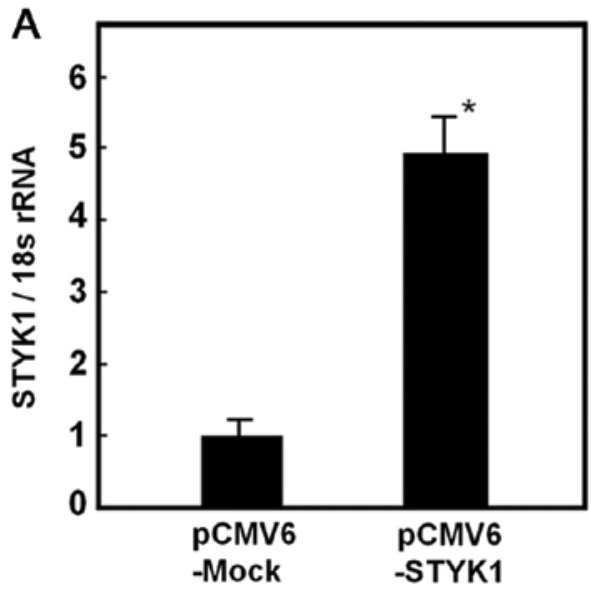

B
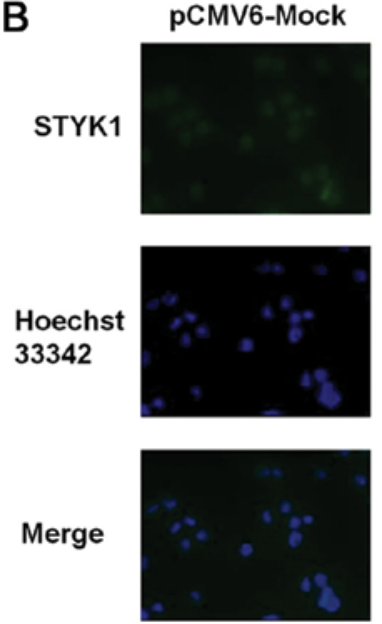

pCMV6-STYK1
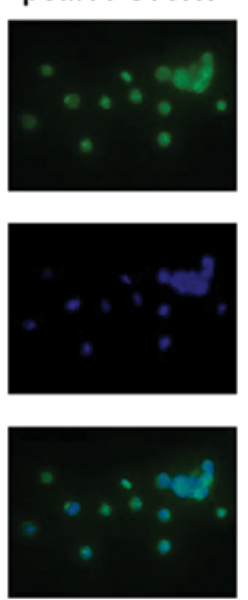

Figure 1. Alteration of (A) STYK1 mRNA and (B) STYK1 protein expression in HL-60 cells transfected with pCMV6-Mock and pCMV6-STYK1. The expression of STYK1 mRNA is presented relative to that of $18 \mathrm{~S}$ rRNA. ${ }^{*} \mathrm{p}<0.0001$ by Student's t-test.

Caspase activity. Caspase-3 and -7 activities were measured using Caspase-Glo 3/7 assay kit (Promega, Madison, WN, USA). Cells transfected with control vector or STYK1 were plated onto 96-well plates (Costar, Corning, NY, USA), and were then treated with doxorubicin and etoposide. After 24and 48-h incubations, $100 \mu \mathrm{l}$ of the assay reagent was added to each well, and incubated for $1 \mathrm{~h}$ at RT. The intensity of luminescence signal (RLU, relative light units) was then measured using a Veritas ${ }^{\mathrm{TM}}$ microplate luminometer (Promega).

Measurement of cell viability. Cells were plated in 96-well plates at a density of 20,000 cells per well in RPMI supplemented with $10 \%$ FBS. The cells were incubated for appropriate period, and were then analyzed using the Cell Titer-Glo ${ }^{\mathrm{TM}}$ Luminescent Cell Viability Assay (Promega) according to the manufacturer's instructions. The level of ATP-driven luminescence signal (RLU, relative light units), which correlates with the number of viable cells, was measured using Veritas microplate luminometer.

Cell cycle analysis. Cells plated onto 6-well culture dishes (Costar) were washed with FBS-free medium and PBS. The cells were then harvested and fixed with $70 \%$ ethanol at $4^{\circ} \mathrm{C}$. After centrifugation, the cell pellets were treated with RNase A, and stained with $1 \mathrm{ml}$ of hypotonic fluorochrome solution $(50 \mu \mathrm{g} / \mathrm{ml}$ propidium iodide and $0.1 \%$ sodium citrate) at room temperature for $30 \mathrm{~min}$. The cells were then kept on ice, and 20,000 cells/sample were analyzed using a Canto flow cytometer (Becton Dickinson Japan, Tokyo, Japan).

\section{Results}

Alteration in drug sensitivity by transfection of a STYK1 expression vector. To determine the importance of STYKI expression for the drug sensitivity of leukemic cells, we transfected leukemia cells with a STYK1 (pCMV6-STYK1) or control vector (pCMV6-Mock). First, we determined the STYK1 mRNA expression levels in three leukemia cell lines to select the appropriate cells for transfection; the mRNA expression was lowest in HL-60 cells (data not shown), and so these cells were used in subsequent experiments. Three micrograms of either vector were transfected into HL-60 cells, and G418-selected stably expressing cells were obtained. In cells transfected with pCMV6-STYK1, a 4.9-fold increase in STYK1 mRNA was detected compared with cells transfected with pCMV6-Mock (Fig. 1A). STYK1 protein expression was also assessed, and increased expression was detected in pCMV6-STYK1-transfected cells using immunocytochemistry (Fig. 1B).

Stable transfectants were treated with different concentrations of doxorubicin and etoposide, and cultured for 48 h. Cells transfected with pCMV6-STYK1 exhibited significantly lower rates of growth inhibition in response to both drugs compared with pCMV6-Mock-transduced cells (Fig. 2). This altered drug sensitivity was more potent in doxorubicin-treated cells. Interestingly, cells transfected with pCMV6-Mock already had some resistance against etoposide, suggesting some cross-resistance between the selection drug G418 sulfate and etoposide.

Alteration of drug-induced caspase activity and the cell cycle by STYK1 transfection. We next examined whether STYK1 overexpression affected drug-induced caspase activity. Cells transfected with pCMV6-Mock or pCMV6STYK1 were treated with doxorubicin and etoposide for 24 and $48 \mathrm{~h}$; apoptosis was then assessed using caspase assays. Caspase activity was increased markedly by drug treatment in cells transfected with either vector; however, the caspase activity was decreased significantly in cells transfected with pCMV6-STYK1 compared with pCMV6-Mock (Fig. 3). After $48 \mathrm{~h}$ of treatment with doxorubicin and etoposide, the number of cells in the sub-G1 phase was increased in cells transfected with either vector, but the increase was less in cells transfected with pCMV6-STYK1 (Fig. 4).

Microarray analysis of STYK1-regulated genes. To determine the mechanism by which STYK1 induces drug resistance, the expression of $\sim 65,000$ genes contained on a 

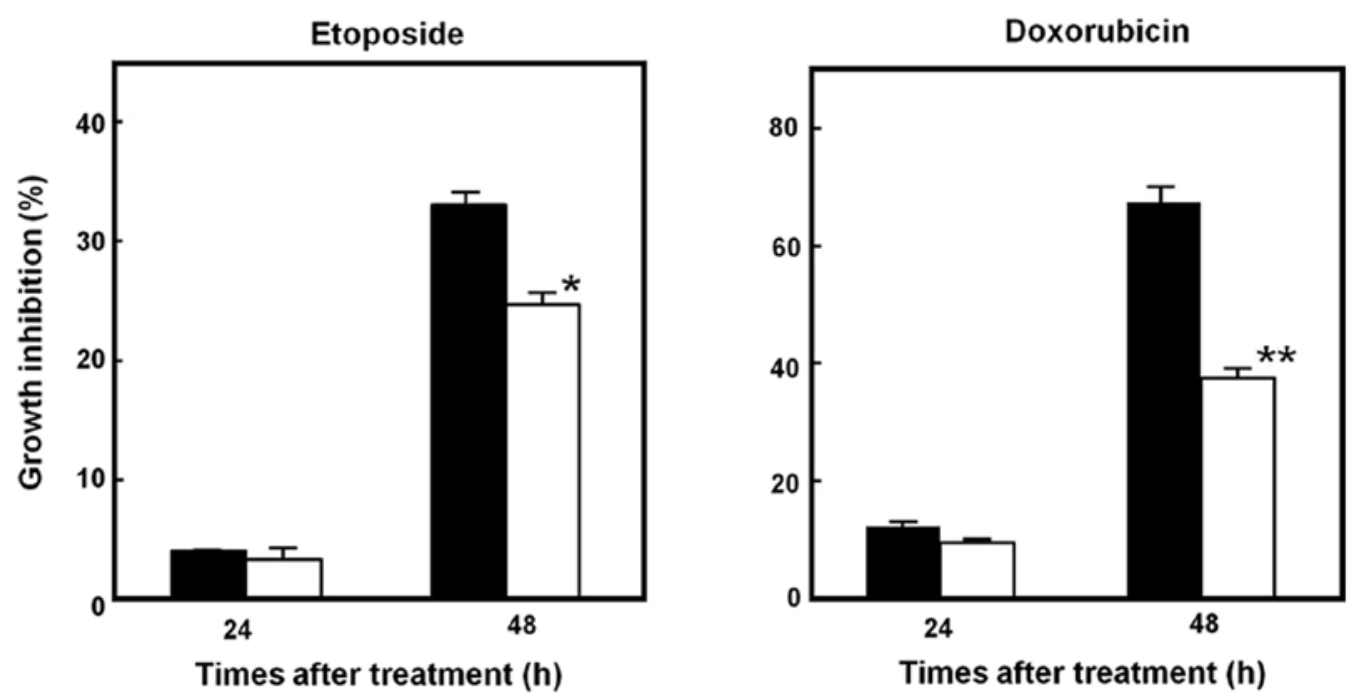

Figure 2. STYK1 overexpression reduced anticancer drug-induced growth inhibition. HL-60 cells transfected with pCMV6-Mock (black bars) and pCMV6-STYK1 (white bars) were treated with etoposide $(1 \mu \mathrm{M})$ and doxorubicin $(0.1 \mu \mathrm{M})$, and cell viability was assessed after 24 and $48 \mathrm{~h}$. Growth inhibition rate (\%) was calculated by relative percentage when the relative light units (RLU) of ATP at 24 and $48 \mathrm{~h}$ was $100 \%$. " $\mathrm{p}<0.015$ and ${ }^{* *} \mathrm{p}<0.0005$ by Student's t-test.
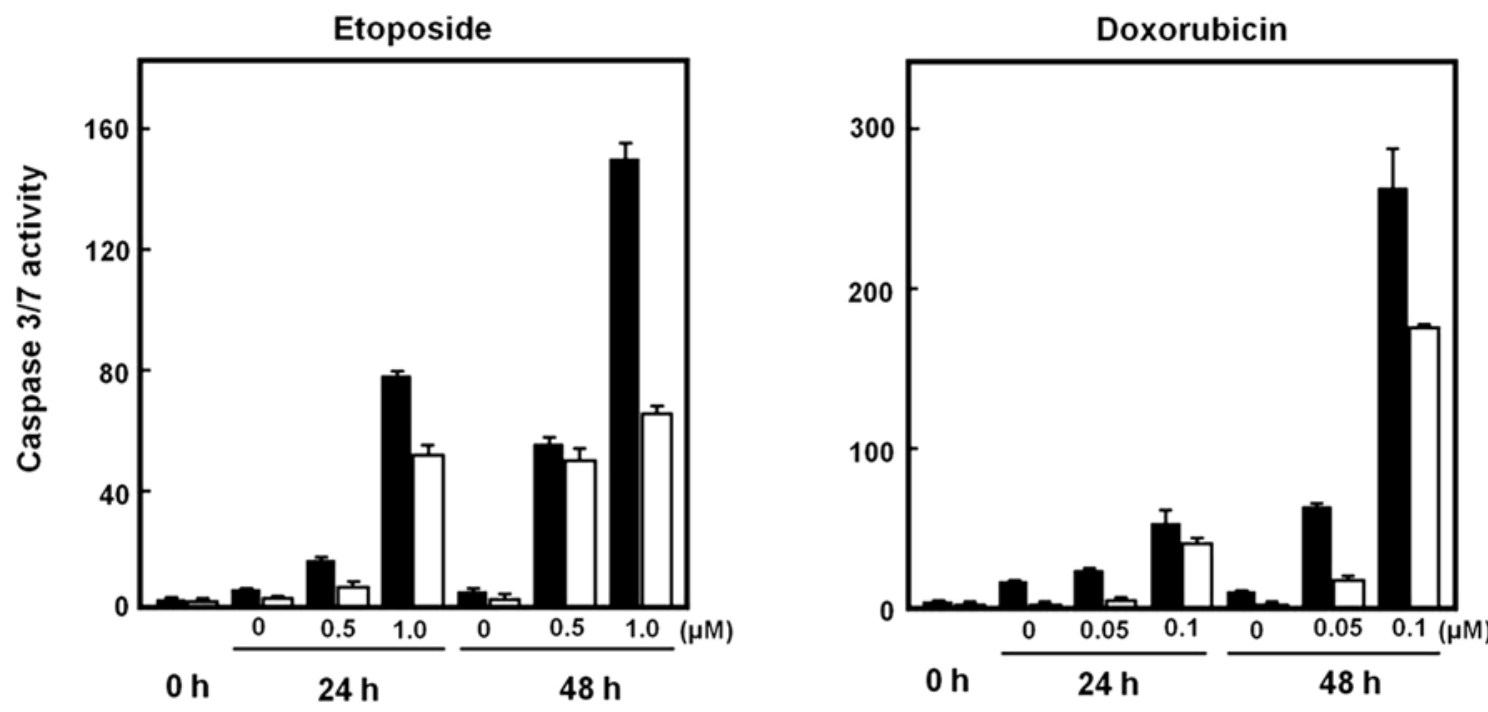

Figure 3. STYK1 overexpression altered anticancer drug-induced caspase activity in HL-60 cells. HL-60 cells transfected with pCMV6-Mock (black bars) and pCMV6-STYK1 (white bars) were treated with etoposide and doxorubicin, and caspase activity was assessed after 24 and $48 \mathrm{~h}$. p $<0.05$ was confirmed in all sets of experiments. The cell number in each sample was counted and caspase activity (RLU, relative light units) is presented as values that have been normalized for cell number.

microarray were analyzed in cells transfected with pCMV6Mock and pCMV6-STYK1. Expression of numerous numbers of molecules was altered and therefore the expression change exceeding 4-fold was taken into consideration for significant alteration. In Table I, we list 11 genes related to cell proliferation or death whose expression was altered $\geq 4$-fold by STYK1 expression. Interestingly, the increased mRNA expression of three different tyrosine kinases was detected. In addition, the expression of $\mathrm{NF}-\kappa \mathrm{B}$-activating and cell death-promoting molecules was inversely regulated, which may contribute to drug resistance. The expression of genes that regulate stem cell renewal, which is particularly important for the maintenance of hematopoietic stem cells, was increased significantly. The altered gene expression detected using microarrays was confirmed using RT-PCR; final analyses revealed that nine genes excepting CARD18 and DAPL1 were apparently upregulated (Fig. 5; the primers used to amplify each gene are shown in Table II).

Comparison of STYK1 mRNA expression in the patients with acute leukemia (Table III). We previously found that STYK1 mRNA is highly expressed in leukemic patients compared to non-leukemic individuals (7). In this study we next examined whether the level of STYK1 mRNA expression before therapy could reflect therapeutic effect of anticancer drugs in leukemic patients. STYK1 mRNA was detected in all the peripheral blood and bone marrow blood samples. When the expression was compared between two groups with 
Table I. Cell proliferation/death-related molecules detected by microarray. ${ }^{\mathrm{a}}$

\begin{tabular}{|c|c|c|c|}
\hline Candidate & $\log 2$ ratio & Expression ratio & Molecular function \\
\hline FGF17 & 3.62 & 12.29 & Proliferation factor \\
\hline FGF20 & 5.00 & 32.00 & Proliferation factor \\
\hline EphA4 & 5.03 & 32.67 & Receptor type tyrosine kinase \\
\hline FLT4 & 2.59 & 6.02 & Receptor type tyrosine kinase \\
\hline STK31 & 6.94 & 122.79 & Receptor type tyrosine kinase \\
\hline MAPK4 & 2.54 & 5.82 & $\mathrm{NF}-\kappa \mathrm{B}$ activation \\
\hline TNF RSF11A & 3.61 & 12.21 & $\mathrm{NF}-\kappa \mathrm{B}$ activation \\
\hline NOV (CCN3) & 5.47 & 44.32 & Stem cell replication \\
\hline SALL4 & 5.79 & 55.33 & Stem cell replication \\
\hline CARD18 & -3.96 & 0.06 & Apoptosis accerelation \\
\hline DAPL1 & -4.39 & 0.05 & Apoptosis accerelation \\
\hline
\end{tabular}

${ }^{\text {a A }}$ 4-fold change was considered to be significant. FGF, fibroblast growth factor; EphA4, ephrin receptor A4; FLT4, fms-related tyrosine kinase 4; STK31, serine/threonine kinase 31; MAPK4, mitogen-activated protein kinase 4; TNF RSF11A, tumor necrosis factor receptor superfamily 11A; NOV, nephroblastoma overexpressed; SALL4, sal-like protein 4; CARD18, caspase recruitment domain family, member 18; DAPL1, death-associated protein-like 1 .

either complete remission (CR) and no-response (NR), NR group exhibited significantly higher expression of $S T Y K 1$ $(3,572 \pm 2,483)$ compared with the CR group $(635 \pm 560$, $\mathrm{p}<0.001$, Fig. 6A). The NR group also showed higher expression in bone marrow samples $(2,257 \pm 699)$ compared with the CR group (577 \pm 416 , Fig. 6B). In both peripheral blood and bone marrow blood samples, this increased expression in NR groups was not specific to individual types of leukemia. Regarding choromosomal abnormality, however, all 5 patients with p53 deletion, Ph1 chromosome, and MLL/ AF4 chimera gene, known as markers for poor response to chemotherapy, showed extremely higher mRNA expression compared with the mean of the CR group. Furthermore, also all peripheral blood and bone marrow blood samples from patients without other prognostic-related factors exhibited higher STYK1 mRNA expression compared with the mean value of the $\mathrm{CR}$ group.

\section{Discussion}

In this study, we provided the first evidence that the tyrosine kinase STYK1 acts as resistance factor against anticancer drugs. In contrast to the previously reported classical receptor protein tyrosine kinases (RPTKs), STYKI lacks an extracellular domain as the receptor for a specific ligand. Therefore, it is thought that growth and survival signals are transmitted through the autoactivation by STYKI expression itself, even in the absence of a specific ligand $(8,9)$. Therefore, we examined whether STYK1 overexpression causes the resistance to anticancer drugs, resulting in a significant decrease in drug sensitivity by inhibiting caspase activity. We then performed microarray analyses to identify candidate STYK1-regulated genes and revealed that other RPTKs were induced by STYK1. The mechanism behind this phenomenon is unclear; however, molecules important for NF- $\mathrm{KB}$ activation were also induced. This suggests that several tyrosine kinases could cooperatively elicit cell survival signals to stimulate drug resistance.
Table II. Primer sequences for RT-PCR.

\begin{tabular}{ll}
\hline Target molecules & \multicolumn{1}{c}{ Primer sequence $\left(5^{\prime} \rightarrow 3^{\prime}\right)$} \\
\hline FGF17-F & AGCTGCTGATTCTCTGCTGTCA \\
FGF17-R & GCTCAGCTGGTCGGTCATG \\
FGF20-F & CGCAGGTATTTTGTGCACTT \\
FGF20-R & TCCACTGGTCTAGGTAAGAAATGTGT \\
EphA4-F & AAGGATCAGAATGAGCGAAGCT \\
EphA4-R & CGCACGTGGAAAACATAGGA \\
FLT4-F & GGCTTCACCATCGAATCCAA \\
FLT4-R & CCAGCGCAGATGCTCGTA \\
STK31-F & CTTGCTTCCAGAACTGACATCTGT \\
STK31-R & GATCTATGCCCCCACAAAGGA \\
MAPK4-F & ACTGCTCCTTTCCCCAATAA \\
MAPK4-R & CCCCAGCAAAGAGCATTCTC \\
TNF RSF11A-F & GGTCAGCAGGGAGCATGTG \\
TNF RSF11A-R & CCCTGACAGACACCACCTTGA \\
NOV-F & AAATTTCAGCCAAGCTGCAAA \\
NOV-R & CAGTTAGGCTCAGGCAGTAGCA \\
SALL4-F & AAGTGTAAGGGTCGGAGCAGTCT \\
SALL4-R & AATGTCGAGGGTCCCACAAA \\
CARD18-F & TGGATAAGGCTCGAGTCTTGATT \\
CARD18-R & GGCAAGTTGAGGGTCTTCTTCA \\
DAPL1-F & AGAAATTGGCACCTTGGAAAGA \\
DAPL1-R & CATTCAGGGCATCCAGTGTCT \\
\hline
\end{tabular}

F, forward; R, reverse; FGF, fibroblast growth factor; EphA4, ephrin receptor A4; FLT4, fms-related tyrosine kinase 4; STK31, serine/threonine kinase 31; MAPK4, mitogen-activated protein kinase 4; TNF RSF11A, tumor necrosis factor receptor superfamily 11A; NOV, nephroblastoma overexpressed; SALL4, sal-like protein 4; CARD18, caspase recruitment domain family, member 18; DAPL1, death-associated protein-like 1 . 

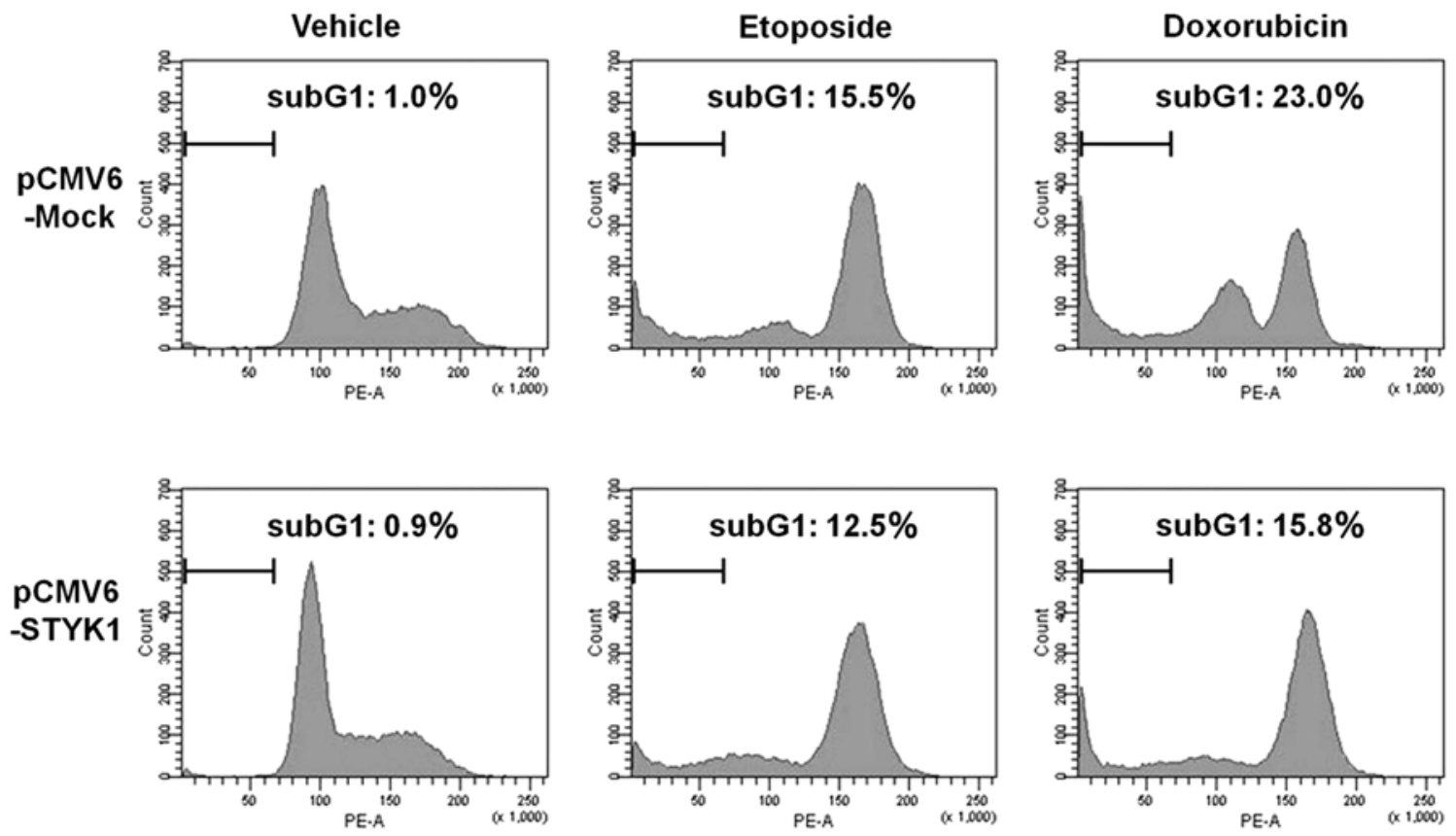

Figure 4.STYK1 overexpression altered anticancer drug-induced cell cycle patterns in HL-60 cells. HL-60 cells transfected with pCMV6-Mock and pCMV6-STYK1 were treated with etoposide $(1 \mu \mathrm{M})$ and doxorubicin $(0.1 \mu \mathrm{M})$; cell cycle analysis was performed after $48 \mathrm{~h}$.

\begin{tabular}{|c|c|c|c|c|c|c|c|c|c|c|c|}
\hline \multicolumn{2}{|c|}{ FGF17 } & \multicolumn{2}{|c|}{ FGF20 } & \multicolumn{2}{|c|}{ EphA4 } & \multicolumn{2}{|c|}{ FLT4 } & \multicolumn{2}{|c|}{ STK31 } & \multicolumn{2}{|c|}{$18 \mathrm{~S}$} \\
\hline 1 & 2 & 1 & 2 & 1 & 2 & 1 & 2 & 1 & 2 & 1 & 2 \\
\hline 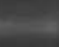 & -6 & 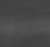 & 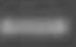 & & 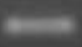 & 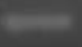 & 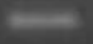 & 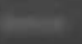 & 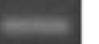 & $=$ & $=$ \\
\hline \multicolumn{2}{|c|}{ MAPK4 } & \multicolumn{2}{|c|}{ TNF RSF11A } & \multicolumn{2}{|c|}{ NOV } & \multicolumn{2}{|c|}{ SALL4 } & \multicolumn{2}{|c|}{ CARD18 } & \multicolumn{2}{|c|}{ DAPL1 } \\
\hline 1 & 2 & 1 & 2 & 1 & 2 & 1 & 2 & 1 & 2 & 1 & 2 \\
\hline & 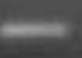 & & + & & - & & - & $=$ & 8.5 & & $x$ \\
\hline
\end{tabular}

Figure 5. RT-PCR and microarray analysis of the expression of candidate STYK1-regulated genes. PCR was performed after cDNA synthesis; samples were then analyzed by electrophoresis on $2 \%$ agarose gels (100 V, 60 min). 1, pCMV6-Mock; 2, pCMV6-STYK1.
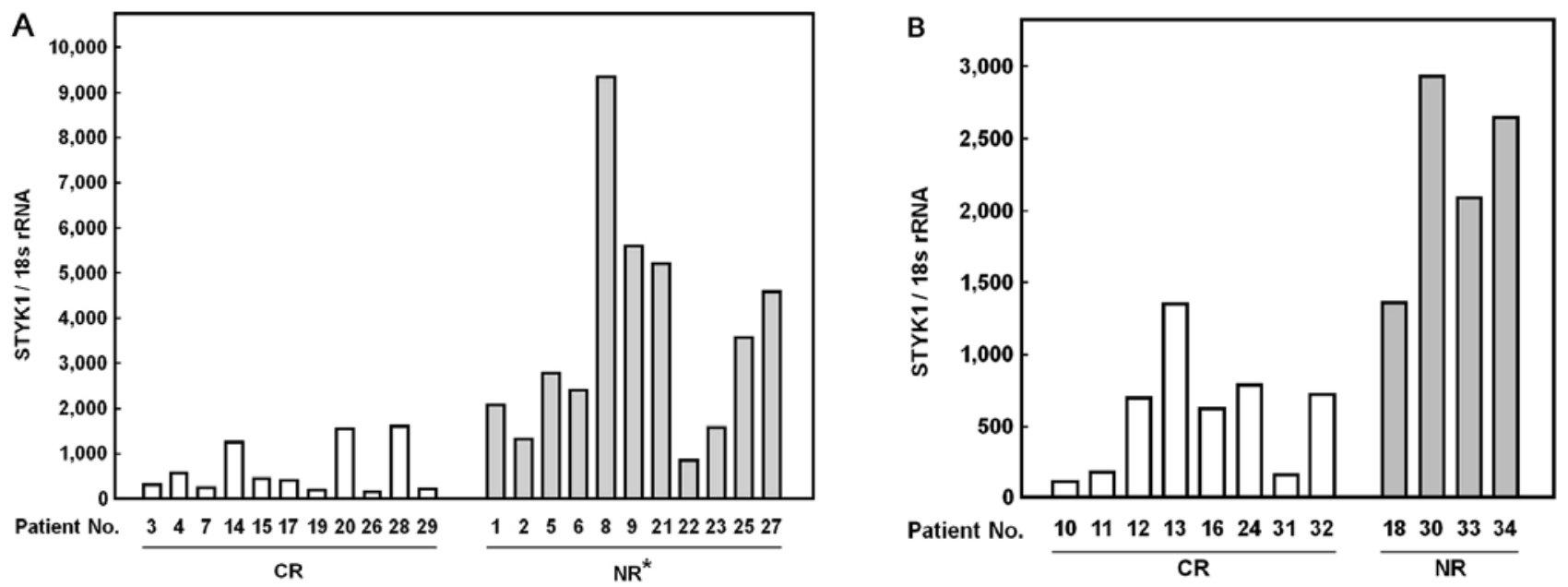

Figure 6. Comparison of the expression of $S T Y K 1$ mRNA in (A) peripheral blood cells and (B) bone marrow blood cells from acute leukemia patients in the complete remission (CR) or non-responder (NR) groups. The expression of STYK1 mRNA was measured using quantitative RT-PCR and the results are presented relative to the expression of $18 \mathrm{~S}$ rRNA. ${ }^{*} \mathrm{p}<0.001$ by Mann-Whitney rank-sum test. 
Table III. Patient characteristics and STYK1 mRNA expression.

\begin{tabular}{|c|c|c|c|c|c|c|c|}
\hline No. & Type & $\mathrm{A} / \mathrm{C}$ & $\mathrm{PB} / \mathrm{BM}$ & $\mathrm{U} / \mathrm{R}$ & Response & Significant abnormality ${ }^{a}$ & Value \\
\hline 1 & AML M0 & $\mathrm{C}$ & $\mathrm{PB}$ & $\mathrm{U}$ & NR & & 2,079 \\
\hline 2 & AML M1 & A & $\mathrm{PB}$ & $\mathrm{U}$ & NR & & 1,334 \\
\hline 3 & AML M2 & $\mathrm{A}$ & $\mathrm{PB}$ & $\mathrm{R}$ & $\mathrm{CR}$ & & 319 \\
\hline 4 & AML M2 & $\mathrm{C}$ & $\mathrm{PB}$ & $\mathrm{R}$ & $\mathrm{CR}$ & & 579 \\
\hline 5 & AML M2 & $\mathrm{C}$ & $\mathrm{PB}$ & $\mathrm{R}$ & NR & $\mathrm{t}(8 ; 21)$ & 2,761 \\
\hline 6 & AML M2 & $\mathrm{C}$ & $\mathrm{PB}$ & $\mathrm{R}$ & NR & & 2,387 \\
\hline 7 & AML M2 & $\mathrm{A}$ & PB & $\mathrm{U}$ & $\mathrm{CR}$ & $\mathrm{t}(8 ; 21)$ & 262 \\
\hline 8 & AML M2 & $\mathrm{A}$ & $\mathrm{PB}$ & $\mathrm{R}$ & NR & p53 deletion & 9,345 \\
\hline 9 & AML M2 & A & $\mathrm{PB}$ & $\mathrm{R}$ & NR & add 12.p11 & 5,585 \\
\hline 10 & AML M2 & A & $\mathrm{BM}$ & $\mathrm{U}$ & $\mathrm{CR}$ & & 115 \\
\hline 11 & AML M2 & A & $\mathrm{BM}$ & $\mathrm{U}$ & $\mathrm{CR}$ & & 180 \\
\hline 12 & AML M2 & A & $\mathrm{BM}$ & $\mathrm{U}$ & $\mathrm{CR}$ & & 695 \\
\hline 13 & AML M2 & $\mathrm{A}$ & $\mathrm{BM}$ & $\mathrm{U}$ & $\mathrm{CR}$ & inv 16 & 1,345 \\
\hline 14 & AML M3 & A & $\mathrm{PB}$ & $\mathrm{U}$ & $\mathrm{CR}$ & $\mathrm{t}(15 ; 17)$ & 1,254 \\
\hline 15 & AML M3 & A & $\mathrm{PB}$ & $\mathrm{U}$ & $\mathrm{CR}$ & $t(15 ; 17)$ & 435 \\
\hline 16 & AML M3 & A & $\mathrm{BM}$ & $\mathrm{U}$ & $\mathrm{CR}$ & $t(15 ; 17)$ & 618 \\
\hline 17 & AML M4 & A & $\mathrm{PB}$ & $\mathrm{R}$ & $\mathrm{CR}$ & inv16 & 412 \\
\hline 18 & AML M4 & A & $\mathrm{BM}$ & $\mathrm{U}$ & NR & & 1,355 \\
\hline 19 & AML M6 & A & $\mathrm{PB}$ & $\mathrm{U}$ & $\mathrm{CR}$ & & 174 \\
\hline 20 & AML M6 & A & $\mathrm{PB}$ & $\mathrm{U}$ & $\mathrm{CR}$ & & 1,558 \\
\hline 21 & AMLL & $\mathrm{A}$ & $\mathrm{PB}$ & $\mathrm{U}$ & NR & 21 trisomy, $\mathrm{Ph} 1$ & 5,205 \\
\hline 22 & AMLL & A & $\mathrm{PB}$ & $\mathrm{R}$ & NR & & 865 \\
\hline 23 & AMLL & $\mathrm{A}$ & $\mathrm{PB}$ & $\mathrm{U}$ & NR & & 1,585 \\
\hline 24 & AMLL & $\mathrm{C}$ & $\mathrm{BM}$ & $\mathrm{U}$ & $\mathrm{CR}$ & & 785 \\
\hline 25 & B-ALL & $\mathrm{C}$ & $\mathrm{PB}$ & $\mathrm{U}$ & NR & 21 trisomy & 3,569 \\
\hline 26 & B-ALL & $\mathrm{A}$ & $\mathrm{PB}$ & $\mathrm{R}$ & $\mathrm{CR}$ & & 146 \\
\hline 27 & B-ALL & $\mathrm{C}$ & $\mathrm{PB}$ & $\mathrm{U}$ & NR & $\mathrm{t}(4 ; 11) \mathrm{MLL} / \mathrm{AF} 4$ & 4,580 \\
\hline 28 & T-ALL & A & $\mathrm{PB}$ & $\mathrm{U}$ & $\mathrm{CR}$ & & 1,613 \\
\hline 29 & T-ALL & A & $\mathrm{PB}$ & $\mathrm{U}$ & $\mathrm{CR}$ & & 234 \\
\hline 30 & B-ALL & A & $\mathrm{BM}$ & $\mathrm{U}$ & NR & $\mathrm{Ph} 1$ & 2,940 \\
\hline 31 & B-ALL & $\mathrm{C}$ & $\mathrm{BM}$ & $\mathrm{U}$ & $\mathrm{CR}$ & & 160 \\
\hline 32 & B-ALL & $\mathrm{C}$ & $\mathrm{BM}$ & $\mathrm{U}$ & $\mathrm{CR}$ & & 720 \\
\hline 33 & B-ALL & $\mathrm{C}$ & $\mathrm{BM}$ & $\mathrm{U}$ & NR & 21 tetrasomy & 2,085 \\
\hline 34 & B-ALL & $\mathrm{C}$ & $\mathrm{BM}$ & $\mathrm{U}$ & NR & $\mathrm{t}(8 ; 13), \mathrm{Ph} 1$ & 2,650 \\
\hline
\end{tabular}

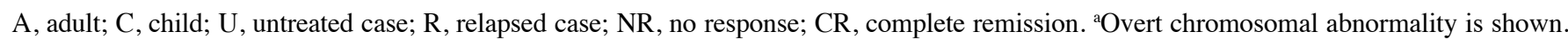
PB, Peripheral blood; BM, Bone marrow.

Interestingly, the expression of the stem cell replicators SALL4 and NOV was induced by STYK1. Both of these genes are expressed at high levels in hematopoietic stem cells $(10,11)$. In addition, Jeong et al demonstrated that the expression of SALL4 is increased in leukemic cells, where it characterizes the leukemic stem cell feature of drug resistance (12). Most tyrosine kinases induce STAT signaling, and it was reported recently that STAT signaling upregulates SALL4 (13). Although the mechanism by which STYK1 augments NOV expression remains unclear, it is possible that STYK1 induces SALL4 expression via STAT signaling. As described above, STYK1 activates the PI3K/Akt pathway (8). This pathway was recently implicated in the induction of multidrug resistance (MDR)-related molecules such as
MDR1 and MRP $(14,15)$. However, the relationship between STYK1 expression and the activation of this pathway is yet to be elucidated.

Based on the above findings, we next analyzed STYK1 gene expression in clinical samples from acute leukemic patients. First of all, regarding STYK1 expression in malignant diseases, we previously demonstrated that $S T Y K 1$ was overexpressed not only in leukemic patients but also in most cancerous tissues from patients with breast and lung cancer, and that its downregulation inhibited the growth of cancer cells $(7,16,17)$. In addition, it was reported that $S T Y K 1$ overexpression could cause the transformation of hematopoietic BaF3 cells (8). Consequently, STYK1 might be involved the acquisition of malignant features of cells. 
The mechanism that regulates $S T Y K 1$ expression is unclear. However, all cases showing the expression of the top 30\% in each peripheral and bone marrow samples (7 peripheral blood and 4 bone marrow samples) formed the NR group, and included all three individuals who had trisomy or tetrasomy of chromosome 21, which is a risk factor for leukemogenesis (Table III). The cancer driver gene RUNX1/AML1 was mapped to chromosome 21 (18); however, the potential relationship between an abnormal chromosome 21 and STYK1 expression has not yet been investigated.

Analysis of leukemic patients revealed that most samples exhibiting high STYK1 expression were in the NR group. Although various molecules such as P-glycoprotein, MRP and GST- $\pi$ stimulate drug resistance, the number of cases in which constitutive expression could be detected before therapy is limited. In the present study, STYKI was highly expressed in the NR group, regardless of the type of leukemia. Furthermore, STYK1 was highly expressed in the NR group of both patients with previously known factors for poor prognosis, and those without any known prognostic factors. This suggests that measuring STYK1 mRNA expression could be a useful general marker for predicting the therapeutic response of various types of acute leukemia. Recently, we found that decreased $S T Y K 1$ mRNA leads to a remarkable growth inhibition of leukemic cells (7). Taken together with the findings in this study, these data suggest that $S T Y K 1$ may be a therapeutic target for patients showing STYK1 overexpression when ideal response to conventional 1st line chemotherapy could not be expected, and actually obtained.

\section{Acknowledgements}

Grants from 'Japan Society for the Promotion of Science' supported this study.

\section{References}

1. Nakao M, Yokota S, Iwai T,Kaneko H, Horiike S, Kashima K, et al: Internal tandem duplication of the flt 3 gene found in acute myeloid leukemia. Leukemia 10: 1911-1918, 1996.

2. Gambacorti-Passerini CB, Gunby RH, Piazza R, Galietta A, Rostagno R and Scapozza L: Molecular mechanisms of resistance to imatinib in Philadelphia-chromosome-positive leukaemias. Lancet Oncol 4: 75-85, 2003.

3. Paietta E, Ferrando AA, Neuberg D, et al: Activating FLT3 mutations in CD117/KIT(+) T-cell acute lymphoblastic leukemias. Blood 104: 558-560, 2004.
4. Yanada M, Takeuchi J, Sugiura I, et al: High complete remission rate and promising outcome by combination of imatinib and chemotherapy for newly diagnosed BCR-ABL-positive acute lymphoblastic leukemia: a phase II study by the Japan Adult Leukemia Study Group. J Clin Oncol 24: 460-466, 2006.

5. Wang YY, Zhou GB, Yin T, et al: AML1-ETO and C-KIT mutation/overexpression in $\mathrm{t}(8 ; 21)$ leukemia: implication in stepwise leukemogenesis and response to Gleevec. Proc Natl Acad Sci USA 102: 1104-1109, 2005.

6. George P, Bali P, Cohen P, et al: Cotreatment with 17-allylaminodemethoxygeldanamycin and FLT-3 kinase inhibitor PKC412 is highly effective against human acute myelogenous leukemia cells with mutant FLT-3. Cancer Res 64: 3645-3652, 2004.

7. Kondoh T, Kobayashi D, Tsuji N, Kuribayashi K and Watanabe N: Overexpression of serine threonine tyrosine kinase 1 /novel oncogene with kinase domain mRNA in patients with acute leukemia. Exp Hematol 37: 824-830, 2009.

8. Liu L, Yu XZ, Li TS, et al: A novel protein tyrosine kinase NOK that shares homology with platelet-derived growth factor/fibroblast growth factor receptors induces tumorigenesis and metastasis in nude mice. Cancer Res 64: 3491-3499, 2004.

9. Chen Y, Li YH, Chen XP, et al: Point mutation at single tyrosine residue of novel oncogene NOK abrogates tumorigenesis in nude mice. Cancer Res 65: 10838-10846, 2005.

10. Ma Y, Cui W, Yang J, et al: SALL4, a novel oncogene, is constitutively expressed in human acute myeloid leukemia (AML) and induces AML in transgenic mice. Blood 108: 2726-2735, 2006.

11. Gupta R, Hong D, Iborra F, Sarno S and Enver T: NOV (CCN3) functions as a regulator of human hematopoietic stem or progenitor cells. Science 316: 590-593, 2007.

12. Jeong HW, Cui W, Yang Y, Lu J, He J, Li A, Song D, Guo Y, Liu BH and Chai L: SALL4, a stem cell factor, affects the side population by regulation of the ATP-binding cassette drug transport genes. PLoS One 6: e18372, 2011.

13. Bard JD, Gelebart P, Amin HM, Young LC, Ma Y and Lai R: Signal transducer and activator of transcription 3 is a transcriptional factor regulating the gene expression of SALL4. FASEB J 23: 1405-1414, 2009.

14. Tazzari PL, Cappellini A, Ricci F, et al: Multidrug resistanceassociated protein 1 expression is under the control of the phosphoinositide 3 kinase/Akt signal transduction network in human acute myelogenous leukemia blasts. Leukemia 21: 427-438, 2007.

15. Chiarini F, Del Sole M, Mongiorgi S, et al: The novel Akt inhibitor, perifosine, induces caspase-dependent apoptosis and downregulates P-glycoprotein expression in multidrug-resistant human T-acute leukemia cells by a JNK-dependent mechanism. Leukemia 22: 1106-1116, 2008.

16. Moriai R, Kobayashi D, Amachika T, Tsuji N and Watanabe N: Diagnostic relevance of overexpressed NOK mRNA in breast cancer. Anticancer Res 26: 4969-4973, 2006.

17. Amachika T, Kobayashi D, Moriai R, Tsuji N and Watanabe N: Diagnostic relevance of overexpressed mRNA of novel oncogene with kinase-domain (NOK) in lung cancers. Lung Cancer 56: 337-340, 2007.

18. Harbott J, Viehmann S, Borkhardt A, Henze G and Lampert F: Incidence of TEL/AML1 fusion gene analyzed consecutively in children with acute lymphoblastic leukemia in relapse. Blood 90: 4933-4937, 1997. 